\title{
Semen quality and waiting time to pregnancy explored using Association Mining
}

Herrera, Jose Alejandro Romero; Bang, Anne Kirstine; Priskorn, Lærke; Izarzugaza, Jose M.G.; Brunak, Søren; Jørgensen, Niels

\section{Published in:}

Andrology

Link to article, DOI:

10.1111/andr.12924

Publication date:

2021

Document Version

Peer reviewed version

Link back to DTU Orbit

Citation (APA):

Herrera, J. A. R., Bang, A. K., Priskorn, L., Izarzugaza, J. M. G., Brunak, S., \& Jørgensen, N. (2021). Semen quality and waiting time to pregnancy explored using Association Mining. Andrology, 9(2), 577-587.

https://doi.org/10.1111/andr.12924

\section{General rights}

Copyright and moral rights for the publications made accessible in the public portal are retained by the authors and/or other copyright owners and it is a condition of accessing publications that users recognise and abide by the legal requirements associated with these rights.

- Users may download and print one copy of any publication from the public portal for the purpose of private study or research.

- You may not further distribute the material or use it for any profit-making activity or commercial gain

- You may freely distribute the URL identifying the publication in the public portal

If you believe that this document breaches copyright please contact us providing details, and we will remove access to the work immediately and investigate your claim 
DR NIELS JøRGENSEN (Orcid ID : 0000-0003-4827-0838)

Article type : Original Article

\section{Title:}

Semen quality and waiting time to pregnancy explored using Association Mining

\section{Authors:}

Jose Alejandro Romero Herrera ${ }^{1}$, Anne Kirstine Bang 2 , Lærke Priskorn², Jose M.G. Izarzugaza ${ }^{3}$, Søren Brunak ${ }^{1,3}$ and Niels Jørgensen ${ }^{2}$

1. NNF Center for Protein Research, University of Copenhagen, Faculty of Health and Medical Sciences, Blegdamsvej 3B, DK-2200 Copenhagen, Denmark.

2. University Department of Growth and Reproduction and International Center for Research and Research Training in Endocrine Disruption of Male Reproduction and Child Health (EDMaRC), Rigshospitalet, Copenhagen University Hospital, DK-2100 Copenhagen, Denmark.

3. Department of Health Technology, Technical University of Denmark, Kemitorvet, DK-2800 Kongens Lyngby, Denmark.

\section{Short title:}

Association Mining: Semen quality and time to pregnancy

\section{Key words:}

Association mining, time to pregnancy, semen quality, age

This article has been accepted for publication and undergone full peer review but has not been through the copyediting, typesetting, pagination and proofreading process, which may lead to differences between this version and the Version of Record. Please cite this article as doi: 10.1111/ANDR.12924

This article is protected by copyright. All rights reserved 


\section{Corresponding author:}

Niels Jørgensen

University Department of Growth and Reproduction

Rigshospitalet, section 5064

Blegdamsvej 9

DK-2100 Copenhagen

Denmark

Tel: +4535455064

Fax: +45 35456054

E-mail: Niels.Joergensen@regionh.dk

This article is protected by copyright. All rights reserved 


\section{Abstract}

Background: Assessment of semen quality is a key pillar in the evaluation of men from infertile couples. Usually, semen parameters are interpreted individually because the interactions between parameters are difficult to account for.

Objectives: To determine how combinations of classical semen parameters and female partner age were associated with waiting time to pregnancy (TTP).

Materials and methods: Semen results of 500 fertile men, information of TTP and partner age were used for regressions and to detect breaking points. For a modified Association Rule Mining algorithm semen parameters were categorized as High, Medium and Low.

Results: Men $\leq 32.1$ years and women $\leq 32.9$ years had shorter TTP than older. Decreasing TTP was associated with increasing level of individual semen parameters up to threshold values: sperm concentration $46 \mathrm{mill} / \mathrm{ml}$, total sperm count 179 mill, progressive motility 63\% and normal morphology $11.5 \%$. Using association mining approximately 100 combinations of semen parameters and partner age were associated to TTP. TTP $\leq 1$ month often co-occurred with high percentages of progressive motility $(\geq 62 \%)$ and morphologically normal spermatozoa $(\geq 10.5 \%)$. Furthermore, TTP $\leq 1$ did not tend to appear with lower percentages of these two semen parameters or high partner age ( $\geq 32$ years). However, high percentages of motile or normal spermatozoa could not compensate for sperm concentration $\leq 42$ or total sperm count $\leq 158$ mill. The prolonging effect of high partner age could not be compensated for by the man's semen quality.

Conclusion: Using association mining we observed that TTP was best predicted when combinations of semen parameters were accounted for. Sperm counts, motility and morphology were all important and no single semen parameter was inferior. Additionally, female age above 32 years had a negative impact on TTP that could not be compensated for by high semen parameters of the man.

This article is protected by copyright. All rights reserved 


\section{Introduction}

Assessment of semen quality is a cornerstone in the evaluation of men from couples having a fertility problem, and determination of the classical semen variables such as sperm concentration, total sperm count, progressive motility and morphology has long been used as predictive markers of fecundity ${ }^{1-4}$. Although semen variables are, in general, correlated, it is a clinical well-known fact that some men present with an apparent perfectly normal level of one parameter (e.g. sperm count) while other parameters such as progressive motility or morphology are impaired. The World Health Organization (WHO) has published reference levels for individual semen parameters based on the $5^{\text {th }}$ percentile of results from 428-1941 fertile men from Europe, Australia or US whose partners had a time-to-pregnancy of up to 12 months $^{4}$. However, in the description of the reference levels the potential impact of interactions between individual semen parameters were not described. Additionally, in the description of the reference levels WHO emphasizes that men whose semen characteristics fall below the lower limits are not necessarily infertile. However, WHO does not mention that semen results above the reference levels does not necessarily ensure a normal fertility potential. Thus, there are studies that show biologically higher thresholds for normal fertility chance than those indicated by the $\mathrm{WHO}^{1-3}$. This shows that semen quality should not be understood as a clearly defined dichotomic phenotype (normal vs. abnormal).

Estimations of sperm concentration, total sperm count, motility and morphology is still basic in the assessment of male reproductive health despite the limited data on the diagnostic value for a couple's ability to conceive. Association Mining has previously been used to detect relations between variables in different kinds of datasets and research areas, such as market basket analysis, web usage mining or bioinformatics ${ }^{5-7}$. For example, Compass is a method for clinical and biobank data mining that employs Self-Organizing Maps and Association Mining ${ }^{8 ; 9}$. Here we used the modified Apriori Association Mining algorithm to uncover statistically significant combinations of semen parameters from fertile men that influence the waiting times for couples to achieve pregnancy (time to pregnancy, TTP).

This article is protected by copyright. All rights reserved 


\section{Materials and Methods}

\section{Study population}

A cohort of 626 men was assembled at the Department of Growth and Reproduction, Copenhagen, Denmark during 1996-1998 $(\mathrm{N}=349)^{10}$ and 2012-2014 $(\mathrm{N}=277)^{11}$. The men were 20-45 years old at the time of inclusion, they were born and raised in Denmark and were residents of the local referral area. They were invited to participate in the study when their female partners were examined routinely during the second trimester of pregnancy. Only natural conceptions - as opposed to fertility treatments - were considered in this study. The men examined in the first period received 500 DKK ( $\sim 67$ Euro) in compensation for their participation, whereas those examined in the last period did not receive any compensation. All men provided signed informed consent before participation.

We excluded 126 men for the current study due to the following reasons: use of contraception at the time of conception ( $\mathrm{N}=61)$, missing TTP data $(\mathrm{N}=9)$, missing results of more than 3 semen parameters $(N=2)$ and ejaculation abstinence lower than 24 hours $(N=29)$. Finally, men from couples with more than 13 months of TTP were not included $(n=25)$ since this is the time when fertility treatment is often started. After all filtering criteria were applied, the study cohort consisted of 500 men.

\section{Questionnaire}

All participants completed a questionnaire prior to examination. It included information on age of the man and the female partner and on time-to-pregnancy. The couple was asked: "Were you or your partner doing anything to avoid pregnancy at the time you became pregnant?". If the answer was "no" the couple was asked: "How many months did it take before you became pregnant? (Thus, having intercourse without doing anything to avoid pregnancy)". Consequently, TTP in this study is defined as the number of months of having unprotected intercourse before conceiving. The men were also asked whether they had previously been responsible for a pregnancy (both life births and abortions) with the current or a previous partner.

This article is protected by copyright. All rights reserved 


\section{Semen analysis}

Semen samples were produced by masturbation in the privacy of a room at the Department of Growth and Reproduction. Semen analysis was conducted as described previously (Jørgensen et al., 2001) and did not vary in the two examination periods. Briefly, semen volume was assessed by weighing assuming a density of $1 \mathrm{~g} / \mathrm{ml}$. For sperm motility assessment, $10 \mu \mathrm{l}$ of well-mixed semen was placed on a glass slide kept at $37^{\circ} \mathrm{C}$, under a $22 \times 22 \mathrm{~mm}$ coverslip, placed on the heated stage of a microscope at $37^{\circ} \mathrm{C}$ and immediately examined at $\times 400$ magnification. Spermatozoa were classified as progressively motile, non-progressively motile, or immotile. For the assessment of sperm concentrations, samples were diluted in a solution of $0.6 \mathrm{~mol} / 1 \mathrm{NaHCO}_{3}$ and $0.4 \%(\mathrm{v} / \mathrm{v})$ formaldehyde in distilled water, and subsequently assessed using a Bürker-Türk haemocytometer (Paul Marienfeld GmbH \& Co. KG, Lauda-Königshofen, Germany). Only spermatozoa with tails were counted. Total sperm count was calculated as semen volume x sperm concentration. Smears were prepared for morphological evaluation, Papanicolaou stained and assessed according to 'stricter criteria' ${ }^{12}$. All assessments were performed in duplicates and represented as a single mean value.

\section{Physical examination}

All participants underwent a physical examination by trained physicians that included determination of testis volume by use of a Prader orchidometer. Body weight and height were measured and used to calculate body mass index (BMI).

\section{Statistics}

General characteristics of the study group are described by their means, standard deviations, medians, $5^{\text {th }}$ and $95^{\text {th }}$ percentiles, or percentages of missing values.

\section{Linear correlation analyses}

Spearman and Pearson correlations and p-values were calculated to measure linear relationships between all variables using the "Hmisc" (version 4.4.0) and "corrplot" (version 0.84) R package. Data were validated by comparing semen quality thresholds for each studied variable against those 
previously described in Bonde et al, Guzick et al, Slama et al ${ }^{1-3}$ by using locally estimated scatterplot smoothing (LOESS) regressions ${ }^{13}$ (for details, see Supplementary Materials and Methods). The association between all studied variables and TTP was investigated by multiple linear regression analysis as baseline model.

\section{Compass pre-processing}

To explore how TTP is associated as a dependent variable to the classical semen parameters (semen volume, sperm concentration, total sperm count, morphology and progressive motility) we used the Compass method ${ }^{8}$. Compass was described in 2013 as a new hybrid method that can combine Self-Organizing Maps ${ }^{14}$ and Association Mining ${ }^{15}$ to identify confident associations within clinical data sets. In this study, participants were not clustered using Self-Organizing Maps (SOMs) due to the small study population. Instead, we used an updated version of Compass (2.0) to pre-process and perform Association Mining on the classical semen parameters, and female partner age (years) as independent variables to explain the dependant variable, TTP. Compass and the software used for Association Mining statistical analyses are implemented in the Python programming language (version 2.7).

The specifications of the Compass method suggest that variables with more than $90 \%$ of missing values should be removed, as well as dichotomous variables with more than $90 \%$ of the values being 0 (or $90 \%$ being 1 ) ${ }^{8 ; 9}$. In addition, variables with a Pearson correlation $r>0.9$ should be removed. Since the dataset fulfilled Compass specifications, no further filtering was necessary.

All semen variables, TTP and female partner age were scaled into z-scores, i.e. number of standard deviations the values of a variable are from the mean of said variable. Missing values were ignored for all subsequent analyses. Quantiles corresponding to the mean of a normalised variable $\pm 0.5 \mathrm{SD}$ defined the "High" and "Low" thresholds and values were then labelled as "High" (H), "Low" (L) and "Medium" (M) accordingly in the association mining (Figure 1).

Two sets of thresholds were considered for the definition of TTP levels:

This article is protected by copyright. All rights reserved 
- Model 1: TTP $<=1$ month for the "Low" threshold and TTP $>=3$ months for the "High" threshold corresponding to the thresholds described above.

- Model 2: TTP $<=1$ month for the "Low" threshold and TTP $>=6$ months for the "High" threshold.

Low and High TTP instances are well balanced in model 1 (high TTP $>=3$ months). The high threshold in model 2 (High TTP $>=6$ months) has been imposed to test a higher TTP threshold because 3 months was deemed too short from a biological point of view. Thus, for model 2 there was an imbalance of the High and Low instances of TTP towards shorter TTP (see Supplementary Materials and Methods for details describing this).

\section{Compass association mining}

Compass identifies association rules using a modified Apriori algorithm ${ }^{8: 15}$. The association rules between the features are displayed as shown below:

$$
\text { Antecedent (X):Attribute (H, M or L) } \rightarrow \text { Consequent (Y):Attribute (H, } M \text { or L) }
$$

Variable $A: H \rightarrow$ Variable $B: H$

$($ Variable A:H, Variable $B: H) \rightarrow$ Variable $C: L$

For example, the rule "Total sperm count:L, Normal morphology:H, Female partner age:H $\rightarrow$ TTP:H" would mean that a combination of "Low" total sperm count, "High" normal morphology and "High" partner age leads to "High" TTP values.

The length of a rule can be controlled to limit the amount of combinations between variables (both left and right side of the arrow). For this analysis, a maximum total length of 4 variables was set, which implies 3 antecedents modifying TTP as consequent.

\section{Association mining statistical analysis}

A "Lift" parameter was used to calculate the performance of each rule. Lift was defined as ratio of the observed frequency of a rule to that expected if the antecedents and consequents were independent ${ }^{16}$.

$$
\operatorname{Lift}(X \rightarrow Y)=\frac{P(X \cap Y)}{P(X) \times P(Y)}
$$


Lift values were converted to a binary logarithmic scale $\left(\log _{2}\right)$ for its interpretation. Negative $\log _{2}$ (lift) values represent rules whose antecedent and consequent tend to be substitutes and do not appear together (negative dependence). Positive $\log _{2}($ lift $)$ values represent rules whose antecedents and consequent are dependent on each other, and therefore co-occur more than expected by chance. Rules with an absolute $\log _{2}$ (lift) value below or equal to 0.5 were not considered of interest.

Two-sided Exact Fisher Tests were used to calculate the statistical significance of associations between antecedents and TTP. P-values were adjusted following the Benjamini-Hochberg False Discovery Rate (FDR) procedure ${ }^{17}$. Only rules with an FDR below 0.05 were considered statistically significant.

In addition, we computed the number of times each antecedent appeared together with each TTP category $(\mathrm{H}, \mathrm{M}$ or $\mathrm{L})$ for significant positive and negative dependent rules. The measurement of these frequencies allowed us to assess the relative contribution of a single variable to different TTP outcomes.

\section{Results}

\section{Study population}

General descriptors of the study population of 500 fertile men are shown in Table 1. Male and female partners median ages were 31.4 and 30.3 years, respectively. The median BMI of the men was 23.9, and their testis size (average of both testicles) was $23 \mathrm{ml}$. Their median sperm concentration was $63 \mathrm{mill} / \mathrm{mL}$, the number of normal spermatozoa and progressively moving spermatozoa were $8.5 \%$ and $55 \%$, respectively. The median TTP of the couples was 2 months. Overall, semen quality did not differ in the two examination periods.

\section{Linear regressions}

Spearman correlations between TTP and semen parameters are shown in Figure 2. Only one strong correlation was found; the one relating sperm concentration and total sperm count ( $\mathrm{rho}=0.86)$. TTP 
was weakly correlated with morphology, progressive motility, and female partner age (rho $=-0.15$, -0.15 and 0.13 , respectively). Since sperm concentration and total sperm count were not highly correlated to TTP, none of the variables were removed on the basis that it would be possible to observe distinct patterns relating to different combinations of these variables in the Association Mining analysis. Although Spearman and Pearson correlation values were highly similar (Pearson $\mathrm{r}=0.99$ ), only Spearman correlations are shown due to the very skewed right tail of TTP values and the semen parameters distributions.

Loess regressions are illustrated in Supplementary Figure 1, and thresholds for the impact of each semen quality variable on TTP derived by using the Elbow method are shown in Table 2. These thresholds are well above the WHO reference levels and similar to those described by Bonde et al, Guzick et al and Slama et al. who investigated associations between semen parameters and TTP (See Supplementary text, section 1$)^{1-4}$. Furthermore, men below the age of 32.1 years and women below 32.9 years, respectively, have shorter TTP than older (Table 2).

Table 3 shows coefficients for associations between individual semen parameters and TTP. Adjusted $\mathrm{R}^{2}$ was equal to 0.03316 , with a Residual Standard Error (RSE) of 2.825 corresponding to a $97.35 \%$ error rate. This suggests that combining all parameters in a linear manner poorly predicts TTP.

\section{Compass pre-processing thresholds}

Thresholds derived from quantiles corresponding to the mean of a normalised variable \pm 0.5 times its standard deviation are shown in Table 4. These threshold values were used to categorise the studied parameters into High, Medium and Low attributes and then perform the association mining. Note, that for model 2, there was an imbalance of the number of instances of High and Low categories towards shorter TTP (See Supplementary text, section 2).

This article is protected by copyright. All rights reserved 


\section{Rules of interest}

We detected 86 and 99 statistically significant rules between antecedents for model 1 and model 2, respectively, and 43 of the rules were shared between the models. Lift and adjusted p-values are shown in a volcano plot in Figure 3. Top 10 associations for positive (highest $\log _{2}($ lift)) and negative (lowest $\log _{2}($ lift)) in each model are shown in Tables 5 and 6 .

\section{Positive dependent rules}

45 and 54 statistically significant rules for models 1 (High TTP $\geq 3$ months) and 2 (High TTP $\geq 6$ months), respectively, had $\log _{2}$ (lift) values above $0.5,10$ of them being the same rule. These rules convey combinations between the consequent (Time to Pregnancy) and antecedents (rest of variables) that strongly tend to co-occur. For example: "Total sperm count:H, Normal morphology:M, Motility:L $\rightarrow$ TTP:H" rule indicates that even though the total sperm count is "High", if progressive motility is "Low" and morphology is only "Medium", TTP values will usually be "High".

\section{Positive dependent rules, model 1 (High TTP $>=3$ months)}

Twelve rules had High TTP as a consequent. In 11 cases, High TTP was found either with Low Sperm concentration, Low Total sperm count or Low Motility, regardless of their combination with High Motility (rule 79), Medium Sperm concentration and Medium Normal morphology (rule 52) or Medium Sperm concentration (rule 59). Single antecedents that were most frequently associated with values of High TTP were High Partner age, Low Motility and Medium Normal Morphology ( $42 \%$ each), followed by Low Sperm concentration and Low Total sperm count (33\% each).

On the other hand, 14 rules had Low TTP $(<=1$ month) as an outcome. None of them included High Partner age, Low Total sperm count, Low Normal morphology or Low Motility with only one exception (rule 65), where Low Sperm concentration was found in combination with High Normal morphology and Medium Partner age. Single antecedents that co-occurred most frequently with Low TTP were Medium Partner age and High Motility (43\% each), followed by High Normal morphology and High Total sperm count (36\% each). 
Positive dependent rules, model 2 (High TTP $>=6$ months)

Most of the positive rules for model 2 (39 rules of 54) had High TTP as a consequent. Combinations of Low/Medium Normal Morphology and Motility and High Partner age were common together with Low/Medium Total sperm count or Sperm concentration (rules 75, 58, 70, 63, 27, 29, 30, 32, for example) and, sometimes, High Sperm concentration (rules 53, 54). Single antecedents that most frequently appeared together with High TTP were High Partner age (51.28\%), Low Motility (44\%) and Medium Normal morphology (36\%), followed by High semen volume and Low Sperm concentration (26\% each).

On the other hand, 14 rules had Low TTP $(<=1$ month) as an outcome. These rules were identical to those found in model 1, the Low threshold for TTP being the same for both models. The differences in $\log _{2}($ lift $)$ and p-values are due to the change in the High threshold. Single antecedents that were most frequently associated with Low TTP were as in model 1.

\section{Negative dependent rules}

In total, 41 and 45 statistically significant rules for model 1 and model 2, respectively, had $\log _{2}($ lift $)$ values below $-0.5,10$ of them being the same rule. These rules convey combinations between the consequent (Time to Pregnancy) and antecedents (rest of variables) that strongly tend to substitute each other. For example: "Partner age:H, Sperm concentration:H $\rightarrow$ TTP:H" rule would indicate that "High" sperm concentrations usually do not co-occur with "High" values of Time to pregnancy, even if the age of the partner would be "High".

Negative dependent rules, model 1 (High TTP $>=3$ months)

Twenty-one rules of 41 had High TTP as a consequent. Combinations of High/Medium Motility, Normal morphology, Sperm concentration, and Low/Medium Partner age were found among the antecedents which did not co-occur with High TTP. Low Total sperm count was found once (rule 40) in combination with Medium Semen volume and Partner age. Rather, combinations of High/Medium Total sperm count, Normal morphology and Motility and Low/Medium Partner age

This article is protected by copyright. All rights reserved 
summarize the rules not observed with High TTP. Single antecedents that most frequently did not co-occur with High TTP values were High Normal morphology (48\%), High Motility and High Total sperm count (43\% each), and Low Partner age (38\%).

Seventeen rules had Low TTP ( $\leq 1$ month) as an outcome. In these cases, most of the rules consisted of combinations of Low/Medium Sperm concentration, Total sperm count, Motility and Normal morphology, and High/Medium Partner Age. However, an exception was found for High Total sperm count, which was combined with Low Motility and High Semen volume (rule 18). Single antecedents that most frequently did not co-occur with Low TTP values were High Female partner age (53\%), Low Sperm concentration (47\%), Low Normal morphology (41\%) and Low Motility (35\%).

Negative dependent rules, model 2 ( $>=6$ months)

Out of 45 rules, 16 had High TTP as a consequent. Compared to model 1, all the rules were composed by combinations of High/Medium Total sperm count, Sperm concentration, Morphology, Normal motility, Semen volume and Low/Medium Partner age - no exceptions were found. Single antecedents that most frequently did not co-occur with High TTP values were High Total sperm count (50\%), High Sperm concentration and High Morphology (44\% each).

On the other hand, 17 rules had Low TTP ( $<=1$ month) as an outcome. These rules were identical to those found in model 1 , since the lower threshold for TTP was unchanged.

In all categories, rules for Medium TTP were more heterogenous with different combinations of variables and categories. For more association mining results (See Supplementary text, section 3).

\section{Discussion}

We have presented a novel way to analyse how combinations of the classical semen parameters, sperm counts, motility, and morphology, and the age of a female partner interact with each other,

This article is protected by copyright. All rights reserved 
with the underlying assumption that these various interactions determine waiting time to pregnancy (TTP).

Using Compass association mining we were able to identify statistically significant associations within these combinations. Generally, we did not detect low total sperm count, low motility or low morphology or high partner age associated with short TTPs $(\leq 1$ month). It was also clear that a high percentage of motile or morphologically normal spermatozoa cannot compensate for low number of spermatozoa. That is, even if the spermatozoa are of high quality, low sperm concentration or low total sperm count will increase the risk of higher TTP. Furthermore, both sperm concentration and total sperm count contributed independently to associations to TTP. If one was redundant, we would expect to find many significant rules that combine sperm concentration and total sperm count which was not the case. In addition, most studies have not directly taken into account the age of the female partner as an impact factor in combination with the classical semen parameters ${ }^{18}$. We clearly detected that the partners age is a crucial parameter to consider. For example, we observed that "high" partner age did not co-occur with Low TTP. Furthermore, the prolonging effect of "high" female partner age on TTP could apparently not be compensated for by the man's semen quality.

Semen samples were delivered approximately four months after successful conception. This indirectly implies that semen quality does not vary much for fertile men without any obvious adverse acting factors like current illness, incl. fever as we found associations to TTP. Nevertheless, the associations may have been stronger if we could have obtained a semen sample produced closer to the time of conception. Investigating the associations between individual semen parameters and TTP we detected markedly higher cut-offs than the current WHO reference levels. Thus, our results support the findings by others ${ }^{1-3}$, and also support the statements in the recent andrology consensus report that the 2010 WHO reference levels have reduced sensitivity to identify men with a fecundity problem, as many will be falsely classified as "normal" and a male factor contributing to a couple infertility may be overlooked if interpretations of semen variables only rely on the WHO reference levels ${ }^{19}$.

This article is protected by copyright. All rights reserved 
Despite that semen quality is a combination of several variables, most studies have focussed on individual parameters. Most likely this is because there is no consensus of how the integrated interpretation of semen quality should be done. Some studies have acknowledged this problem and to various degrees tried to include that. Some of us have published a suggestion for combinations based on both the current WHO reference levels and levels from studies where the biological capacity to achieve a pregnancy became affected ${ }^{20}$. Hitherto, the most advanced study ${ }^{2}$, had semen and pregnancy results from both fertile and infertile couples. They elegantly were able to determine "fertile", "intermediate" and "subfertile" cut-offs of semen quality and showed the interaction between the main semen parameters. In line with that, our baseline model showed that combining all classical semen parameters in a linear manner hardly explains the variability of the TTP outcome. On the other hand, our association mining showed that all the classical semen parameters provide information to explain TTP outcomes. We observed that a single parameter is not superior or inferior to the others in predicting fertility chances, meaning that a "high" level of one semen parameter does not compensate for other "low" measurements. Nonetheless, we showed that certain key combinations of semen parameters and partner age were not seen generally among couples with shorter TTP outcomes, such as high partner age, low sperm concentration and low normal morphology. Thus, all semen variables are important and there are no clear-cut levels that can distinguish between "normal vs. abnormal" as it is often done when referring to the WHO reference levels. The interpretation of semen parameters is complex and does not fit the traditional normal vs. abnormal interpretation of results.

One of the advantages of our approach is that it produces a multitude of complex results reflecting the complex associations between semen quality and fertility chances. On the other hand, the complexity of these results also makes it difficult to comprehend all the details and translate them into clinically meaningful conclusions. As a further development, it will be of clinical relevance to develop an algorithm able to identify a patient's attributes and predict which TTP category the patient would belong to. For example, given enough number of participants, one could train a Random Forest algorithm that could possibly classify TTP categories based on association mining 
rule antecedents. However, our results are based on fertile men, who usually produce semen of higher quality and have shorter TTP values. To overcome this bias, it might be necessary to additionally include a broad cohort of men from infertile couples, i.e., men from couples going for fertility treatment, including both ICSI (intracytoplasmic sperm injection/in vitro fertilisation) and IUI (intrauterine insemination), and assume that their TTP values are above 13 months. After exclusion of couples with an obvious female factor, the cohort would cover the entire spectrum of semen quality in infertile men. This addition would likely make TTP predictions more reliable and accurate. Furthermore, including more variables, e.g. intercourse frequency or ejaculation frequency could improve such an algorithm. Increasing ejaculation frequency may reduce sperm concentration and total sperm counts, however the effect seems to attenuate when ejaculation abstinence period is accounted for $^{21}$.

Our approach surpasses common association mining methods that rely in predefined cut-offs of support and confidence to find frequent rules. These methods prune rules prior to their statistical assessment, and likely increase the risk of Type I errors. In our method, we solved this obstacle by testing first all combinations of rules and then adjust their p-values following the False Discovery Rate procedure. In addition, rules are usually explored visually using scatter plots and focusing only on the significance and strength of the correlation. To our knowledge, it is the first time that rules are displayed in volcano plots, where the statistical significance (p-value) is displayed against lift values. This way, we were able to quickly identify rules with a significant effect on TTP. However, even though volcano plots were useful to identify these single combinations, they do not produce a holistic overview of the patterns that drive TTP outcomes. The rules needed manual inspection in order to provide a comprehensive understanding of the results. Further automatic procedures and network analysis are needed in order to ameliorate this issue.

In conclusion, we have provided an integrative overview of the interactions between classical semen parameters and the age of the female partner on TTP outcomes. Association mining and volcano plots are practical tools to explore these interactions, but further improvements are needed to help reach comprehensive, clinical conclusions. All semen parameters can provide valuable 
information and certain combinations with partner age can influence TTP. Obtaining pregnancy within the first month of trying was generally not detected among men with either low total sperm count, low motility or low morphology or with high partner age. Furthermore, the negative effect of female partner age at 32 years or above could apparently not be compensated for by the man's semen quality. In younger women, short TTPs seemed to mainly rely on a high total number of morphologically normal spermatozoa (high sperm count and high percentage of normal forms).

This article is protected by copyright. All rights reserved 


\section{Acknowledgements}

This work was supported by the Novo Nordisk Foundation (grants NNF14CC0001 and NNF17OC0027594), the Innovation Fund Denmark (grants 5184-00102B and 14-2013-04) and the Independent Research Fund Denmark (grant 8020-00218B).

\section{Disclosures}

Authors declare no conflicts of interest.

\section{Authors contribution}

Study design: JARH, LP and NJ

Data acquisition: AKB, LP and NJ

Data analysis: JARH, LP and NJ

Interpretation of data: All authors

Drafted the paper or revised it critically: JARH, LP, JMGI and NJ

Approval of the submitted and final versions: All authors.

This article is protected by copyright. All rights reserved 


\section{Reference List}

(1) Bonde JP, Ernst E, Jensen TK et al. Relation between semen quality and fertility: a population-based study of 430 first-pregnancy planners. Lancet 1998;352:1172-1177.

(2) Guzick DS, Overstreet JW, Factor-Litvak P et al. Sperm morphology, motility, and concentration in fertile and infertile men. N Engl J Med 2001;345:1388-1393.

(3) Slama R, Eustache F, Ducot B et al. Time to pregnancy and semen parameters: a crosssectional study among fertile couples from four European cities. Human Reproduction 2002;17:503-515.

(4) World Health Organization. WHO laboratory manual for the examination and processing of human semen. 5 th ed. 2010.

(5) Agrawal R, Imielinski T, Swami A. Mining Association Rules Between Sets of Items in Large Databases. SIGMOD Rec 1993;22:207-216.

(6) Effective Personalization Based on Association Rule Discovery from Web Usage Data.: 2001.

(7) Naulaerts S, Meysman P, Bittremieux W et al. A primer to frequent itemset mining for bioinformatics. Briefings in Bioinformatics 2015;16:216-231.

(8) Krysiak-Baltyn K, Nordahl PT, Audouze K, Jorgensen N, Angquist L, Brunak S. Compass: a hybrid method for clinical and biobank data mining. J Biomed Inform 2014;47:160-170.

(9) Jiang L, Audouze K, Romero Herrera JA et al. Conflicting associations between dietary patterns and changes of anthropometric traits across subgroups of middle-aged women and men. Clin Nutr 2020;39:265-275.

(10) Jorgensen N, Andersen AG, Eustache F et al. Regional differences in semen quality in Europe. Human Reproduction 2001;16:1012-1019.

This article is protected by copyright. All rights reserved 
(11) Antonio L, Priskorn L, Nordkap L et al. Bone mineral density is preserved in men with idiopathic infertility. Andrology 2020;8:315-322.

(12) Menkveld R, Stander FS, Kotze TJ, Kruger TF, van Zyl JA. The evaluation of morphological characteristics of human spermatozoa according to stricter criteria. Hum Reprod 1990;5:586-592.

(13) Loader C. Local Regression and Likelihood. 1999.

(14) Kohonen.T. Self-organized formation of topologically correct feature maps. Biological Cybernetics 1982;43:59-69.

(15) Ceglar A, Roddick JF. Association Mining. ACM Computing Surveys 2006;38.

(16) Brin S, Motwani R, Ullman JD, Tsur S. Dynamic Itemset Counting and Implication Rules for Market Baske Data. SIGMOD Rec 1997;26:255-264.

(17) Benjamini Y, Hochberg Y. Controlling the False Discovery Rate: A Practical and Powerful Approach to Multiple Testing. Journal of the Royal Statistical Society Series B (Methodological) 1995;57:289-300.

(18) Patel AS, Leong JY, Ramasamy R. Prediction of male infertility by the World Health Organization laboratory manual for assessment of semen analysis: A systematic review. Arab J Urol 2018;16:96-102.

(19) Barratt CLR, Mortimer D, Amer M et al. The current status and future of andrology: A consensus report from the Cairo workshop group. Andrology 2020;8:27-52.

(20) Damsgaard J, Joensen UN, Carlsen E et al. Varicocele Is Associated with Impaired Semen Quality and Reproductive Hormone Levels: A Study of 7035 Healthy Young Men from Six European Countries. Eur Urol 2016;70:1019-1029.

(21) Carlsen E, Petersen JH, Andersson AM, Skakkebaek NE. Effects of ejaculatory frequency and season on variations in semen quality. Fertil Steril 2004;82:358-366.

This article is protected by copyright. All rights reserved 


\section{Figure legends}

Figure 1: Density plots of value distribution after z-sore scaling. The coloured areas of the density plot represent the attribute assignation ( $\mathrm{L}$ as light blue, $\mathrm{M}$ as yellow or $\mathrm{H}$ as red) to each variable after its z-score scaling. ** denotes statistical significance for a Shapiro-Wilk's normality test for a significance level of 0.05 .

Figure 2: Spearman correlation summary of semen variables for fertile men, their partners' ages and time to pregnancy. Positive correlations are in blue colour and negative correlations in red. Intensity of the colour shows the strength of the correlation (the higher the intensity, the stronger the correlation). Correlation coefficients are displayed inside each cell. White cells represent correlation coefficients with p-values $>0.05$.

Figure 3: Volcano plots for association rules found in model 1 (A) and model 2 (B). Logarithm of Lift values is represented in the $\mathrm{X}$-axis, while negative logarithm of the p-values is displayed in the Y-axis. The horizontal dashed black line represents an adjusted p-value threshold of 0.05. The vertical dashed black lines show where Lift $<0.5$ and $>1.5$. Each dot represents an association rule and are coloured orange if they are found to be of interest, and blue otherwise. Arrows and numbers indicate rules of interest that display large absolute values of logarithmic Lift values and high statistical significance.

This article is protected by copyright. All rights reserved 
Table 1. Characteristics of 500 fertile men with known waiting time-to-pregnancy (TTP).

\begin{tabular}{lccc} 
& Mean \pm SD & Median (5-95)* & \% NAs** \\
\cline { 2 - 4 } Age (years) & $32.0 \pm 4.2$ & $31.4(25.9-39.6)$ & 0.0 \\
Partner age (years) & $30.5 \pm 3.70$ & $30.3(24.8-37.1)$ & 4.8 \\
Height (cm) & $183 \pm 6$ & $183(173-195)$ & 1.0 \\
Weight (kg) & $82.0 \pm 11.5$ & $81.0(66.4-101.3)$ & 1.0 \\
BMI & $24.3 \pm 3.0$ & $23.9(20.2-29.5)$ & 1.0 \\
Testis size, mean of two (ml) & $22 \pm 5$ & $23(15-30)$ & 1.2 \\
Ejaculation abstinence (h) & $90 \pm 69$ & $73(36-202)$ & 0.0 \\
Semen volume (ml) & $4.0 \pm 1.7$ & $3.8(1.8-7.1)$ & 0.0 \\
Sperm concentration (mill/ml) & $76 \pm 59$ & $63(11-179)$ & 0.0 \\
Total sperm count (mill) & $291 \pm 237$ & $234(40-781)$ & 0.0 \\
Normal morphology (\%) & $9.0 \pm 4.6$ & $8.5(2.0-17.0)$ & 8.4 \\
Progressive motility (\%) & $54 \pm 15$ & $55(27-77)$ & 0.0 \\
Time to Pregnancy (months) & $3 \pm 3$ & $2(0-9)$ & 0.0 \\
\hline
\end{tabular}

*5-95: 5-95th percentile. ${ }^{* *}$ NA: Percentage of missing values.

Table 2. Thresholds for ages above which TTP increases, and for semen parameters below which TTP increases. Results from this study are shown in comparison with three previously published studies. Results from 302 among the 500 men in this current study were also included in the publication by Slama et al, 2002.

Age, man (years)

Age, female partner (years)

Semen volume (ml)

Sperm concentration (mill/ml)

Total sperm count (millions)

(Progressive) motility (\%)

Normal Morphology (\%)
This study Bonde et Guzick et Slama et al, WHO lower reference al, 1998 al, 2001 2002 level ( $5^{\text {th }}$ percentile)

\begin{tabular}{ccccc}
\hline 32.1 & - & - & - & - \\
32.9 & - & - & - & - \\
3.5 & - & - & None & 1.5 \\
46 & 40 & 48 & 55 & 15 \\
179 & - & - & 145 & 40 \\
63 & $70^{*}$ & $63^{*}$ & None & 32 \\
11.5 & - & 12 & 19 & 4 \\
\hline
\end{tabular}


Table 3. TTP associations for age of female partner and semen variables. Results are regression coefficients and $95 \% \mathrm{Cls}$ between all studied variables and time to pregnancy (TTP).

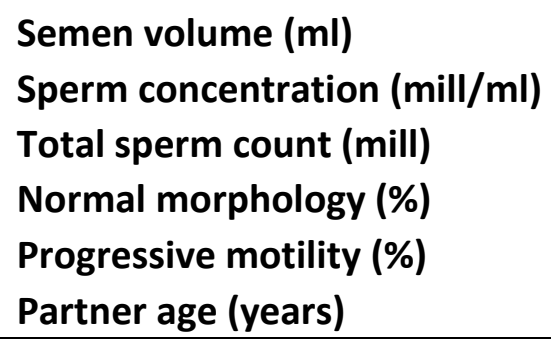

\begin{tabular}{cc} 
Beta $(95 \% \mathrm{Cl})$ & p-value \\
\hline $0.0385(-0.232 ; 0.309)$ & 0.7795 \\
$0.0033(-0.008 ; 0.014)$ & 0.5581 \\
$-0.0009(-0.043 ; 0.002)$ & 0.5434 \\
$-0.0668(-0.131 ;-0.003)$ & $0.011^{*}$ \\
$-0.0242(-0.043 ;-0.006)$ & $0.0397^{*}$ \\
$0.0776(0.005 ; 0.150)$ & $0.0369 *$
\end{tabular}

Beta (95\% Cl): Regression coefficient and 95\% confidence interval. * denotes statistical significance at significance level $\alpha$ of 0.05 .

Table 4. Threshold values that were used to perform association mining and categorize studied parameters into Low ( $\leq$ lower threshold), High ( $\geq$ higher threshold) or Medium. These values represent quantiles corresponding to the median of a variable \pm 0.5 times its standard deviation after z-score normalization.

\begin{tabular}{lccc} 
& Lower threshold & Medium & Higher threshold \\
\cline { 2 - 4 } Time to Pregnancy (months) & $\leq 1$ & $1.1-2.9$ & $\geq 3$ or $\geq 6$ \\
Semen volume (ml) & $\leq 3.2$ & $3.3-4.3$ & $\geq 4.4$ \\
Sperm concentration (mill/ml) & $\leq 42$ & $43-87$ & $\geq 88$ \\
Total sperm count (mill) & $\leq 158$ & $159-317$ & $\geq 318$ \\
Normal morphology (\%) & $\leq 6.9$ & $7.0-10.4$ & $\geq 10.5$ \\
Progressive motility (\%) & $\leq 50$ & $51-61$ & $\geq 62$ \\
Partner age (years) & $\leq 28.6$ & $28.7-31.8$ & $\geq 31.9$
\end{tabular}


Table 5. Top 10 negative (do not tend to co-occur) and Top 10 positive (tend to co-occur) association rules of interest for model 1 (TTP thresholds of 1 and 3 months). Sorted by TTP outcome (High, Low and Medium in that order).

Rule no.

Consequent $\log _{2}($ Lift $) \quad$ Adj. p-value

Top 10 lowest $\log _{2}$ (lift) values (Do not tend to co-occur)

71 Total sperm count:H, Motility:M, Female partner age:L
30 Semen volume:H, Normal morphology:H, Female partner age:M

Semen volume:M, Total sperm count:L, Female partner age:M

Total sperm count:H, Normal morphology:H, Female partner age:L

Total sperm count:M, Motility:H, Normal morphology:M

Motility:H, Normal morphology:M, Female partner age:L

Semen volume:H, Total sperm count:H, Motility:L

Total sperm count:M, Normal morphology:M, Female partner age:H

60 Sperm concentration:L, Total sperm count:M, Normal morphology:L
43 Semen volume:L, Sperm concentration:H, Motility:M

Top 10 highest $\log _{2}$ (lift) values (Tend to co-occur)

16 Semen volume:H, Sperm concentration:L, Motility:H

58 Sperm concentration:L, Total sperm count:M, Motility:H

86 Motility:L, Normal morphology:H, Female partner age:H

15 Semen volume:H, Sperm concentration:H, Female partner age:L

34 Semen volume:M, Sperm concentration: $H$, Motility:L

24 Semen volume:H, Motility: $H$, Female partner age:L

37 Semen volume:M, Total sperm count:H, Motility:L TtP:M

TtP:H $\quad-1.7763 \quad 0.0057$

TtP:H $\quad-1.2167 \quad 0.0024$

TtP:H $\quad-1.1522 \quad 0.0306$

TtP:H $\quad-0.9290 \quad 0.0101$

TtP:H $\quad-0.9290 \quad 0.0284$

TtP:H $\quad-0.9290 \quad 0.0284$

TtP:L $\quad-1.2859 \quad 0.0123$

TtP:L $\quad-1.1036 \quad 0.0334$

TtP:L $\quad-0.9345 \quad 0.0309$

TtP:M $\quad-1.6300 \quad 0.0420$

57 Sperm concentration:M, Normal morphology:L, Female partner age:L

29 Semen volume:H, Normal morphology:H, Female partner age:H

41 Semen volume:M, Motility:L, Female partner age:M

$\begin{array}{lll}\text { TtP:M } & 1.0441 & 0.0050 \\ \text { TtP:M } & 1.0441 & 0.0356 \\ \text { TtP:M } & 1.0441 & 0.0356 \\ \text { TtP:M } & 0.9488 & 0.0290 \\ \text { TtP:M } & 0.9488 & 0.0290 \\ \text { TtP:M } & 0.9044 & 0.0027 \\ \text { TtP:M } & 0.8618 & 0.0429 \\ \text { TtP:M } & 0.8618 & 0.0429 \\ \text { TtP:M } & 0.7928 & 0.0249 \\ \text { TtP:M } & 0.7928 & 0.0249\end{array}$


Table 6. Top 10 negative (do not tend to co-occur) and Top 10 positive association rules of interest for model 2 (TTP thresholds of 1 and 6 months). Sorted by TTP outcome (High, Low and Medium in that order).

Rule no.

Top 10 lowest $\log _{2}$ (lift) values (Do not tend to co-occur)

13 Total sperm count:H, Female partner age:L

51 Sperm concentration:H, Total sperm count:H, Female partner age:L

35 Semen volume:M, Sperm concentration: $H$, Total sperm count:H

21 Normal morphology:H, Female partner age:L

24 Semen volume:H, Total sperm count:H, Motility:L

55 Sperm concentration:M, Total sperm count:H, Normal morphology: $\mathrm{H}$

41 Semen volume:L, Sperm concentration:M, Motility:H

93 Total sperm count:L, Normal morphology:M, Female partner age:L

38 Semen volume:M, Normal morphology:M, Female partner age:L

77 Sperm concentration:L, Normal morphology:M, Female partner age:L
Consequent

Adj. p-value

Top 10 highest $\log _{2}$ (lift) values (Tend to co-occur)

92 Total sperm count:L, Normal morphology:H, Female partner age:H

66 Sperm concentration:L, Total sperm count:M, Motility:L

57 Sperm concentration:M, Total sperm count:L, Normal morphology:M

22 Semen volume:H, Sperm concentration:L, Motility:L

28 Semen volume:H, Motility:M, Normal morphology:M

53 Sperm concentration:H, Motility:L, Normal morphology:L

70 Sperm concentration:L, Motility:M, Normal morphology:M

75 Sperm concentration:L, Normal morphology:H, Female partner age:H

54 Sperm concentration:H, Motility:L, Female partner age:H

58 Sperm concentration:M, Total sperm count:L, Female partner age:H

$\begin{array}{ccc}\text { TtP:H } & -2.1014 & 0.0018 \\ \text { TtP:H } & -1.7478 & 0.0178 \\ \text { TtP:H } & -1.2958 & 0.0194 \\ \text { TtP:H } & -1.2131 & 0.0088 \\ \text { TtP:L } & -1.2859 & 0.0123 \\ \text { TtP:M } & -1.6269 & 0.0166 \\ \text { TtP:M } & 1.4446 & 0.0497 \\ \text { TtP:M } & -1.4446 & 0.0022 \\ \text { TtP:M } & -1.3933 & 0.0037 \\ \text { TtP:M } & -1.3392 & 0.0063\end{array}$

$\begin{array}{lcc}\text { TtP:H } & 1.4122 & 0.0023 \\ \text { TtP:H } & 1.3232 & 0.0013 \\ \text { TtP:H } & 1.2787 & 0.0052 \\ \text { TtP:H } & 1.0556 & 6.00 \mathrm{E}-04 \\ \text { TtP:H } & 1.0556 & 0.0342 \\ \text { TtP:H } & 1.0556 & 0.0342 \\ \text { TtP:H } & 1.0556 & 0.009 \\ \text { TtP:H } & 1.0556 & 0.0342 \\ \text { TtP:H } & 0.9602 & 0.0276 \\ \text { TtP:H } & 0.9602 & 0.0276\end{array}$


Total sperm count -

Sperm concentration
Semen volume

Normal morphology

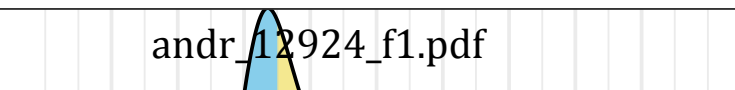




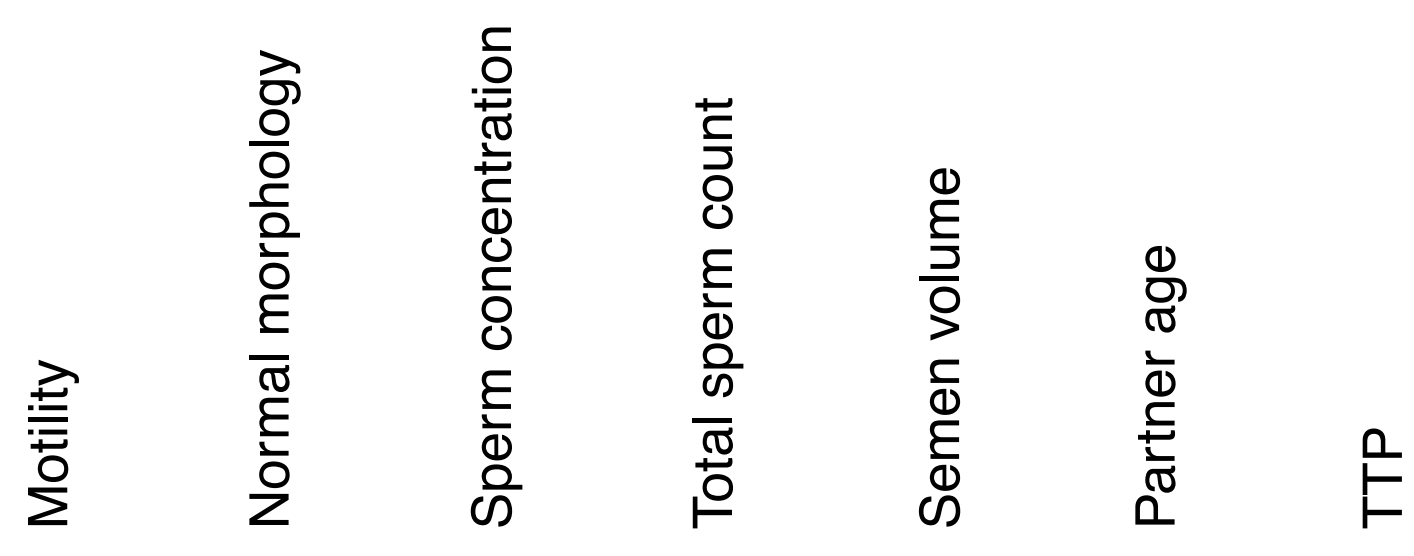

Normal morphology
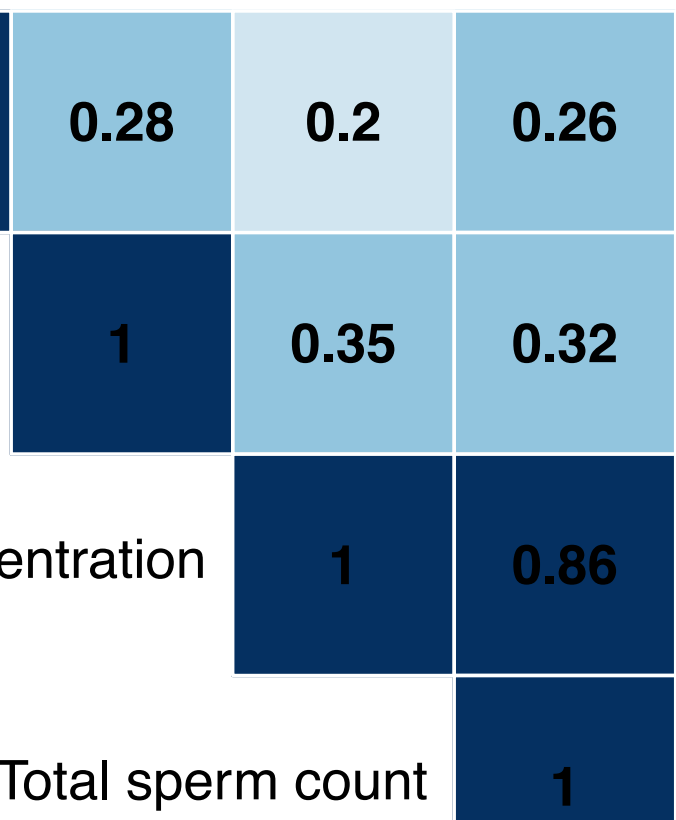

Total sperm count

Sperm concentration
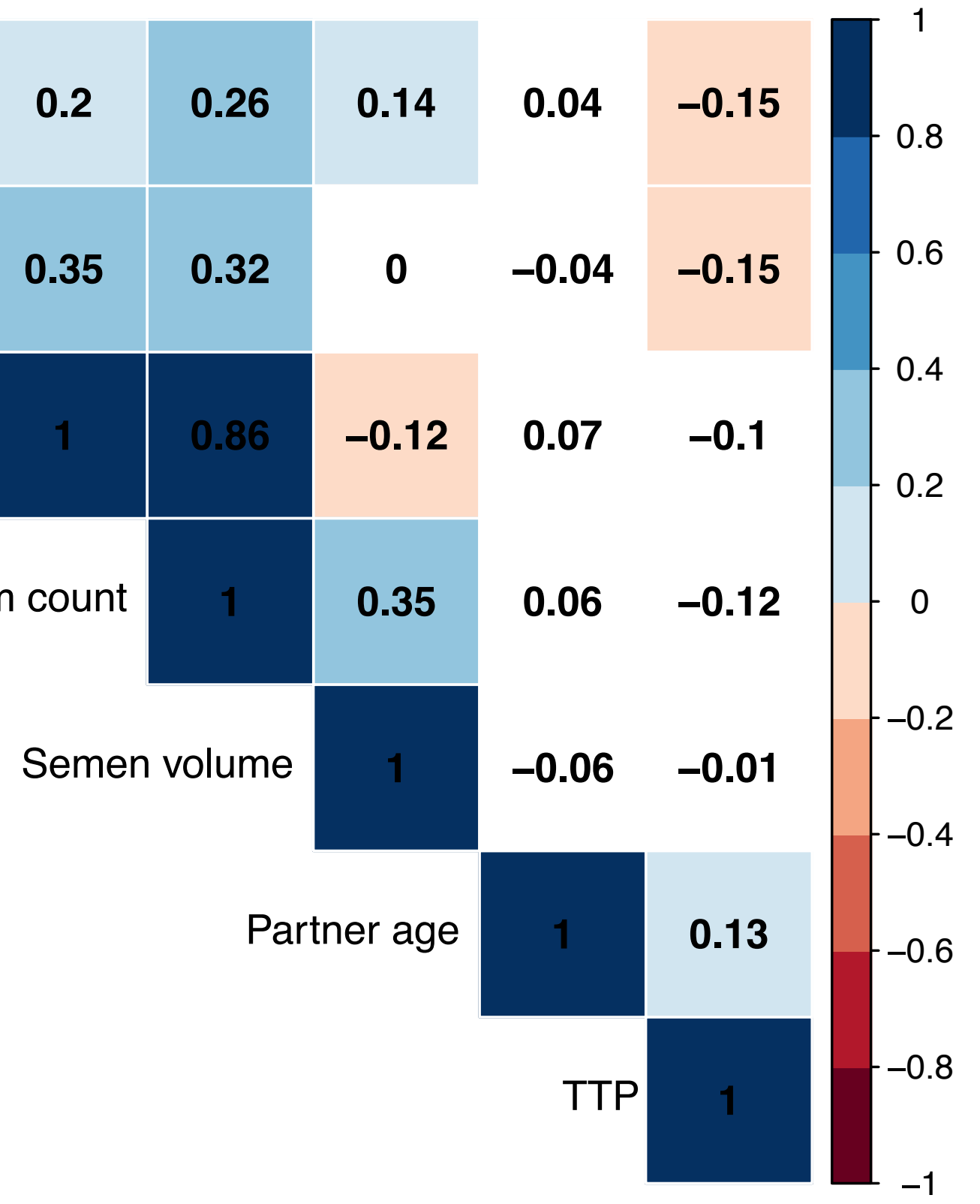

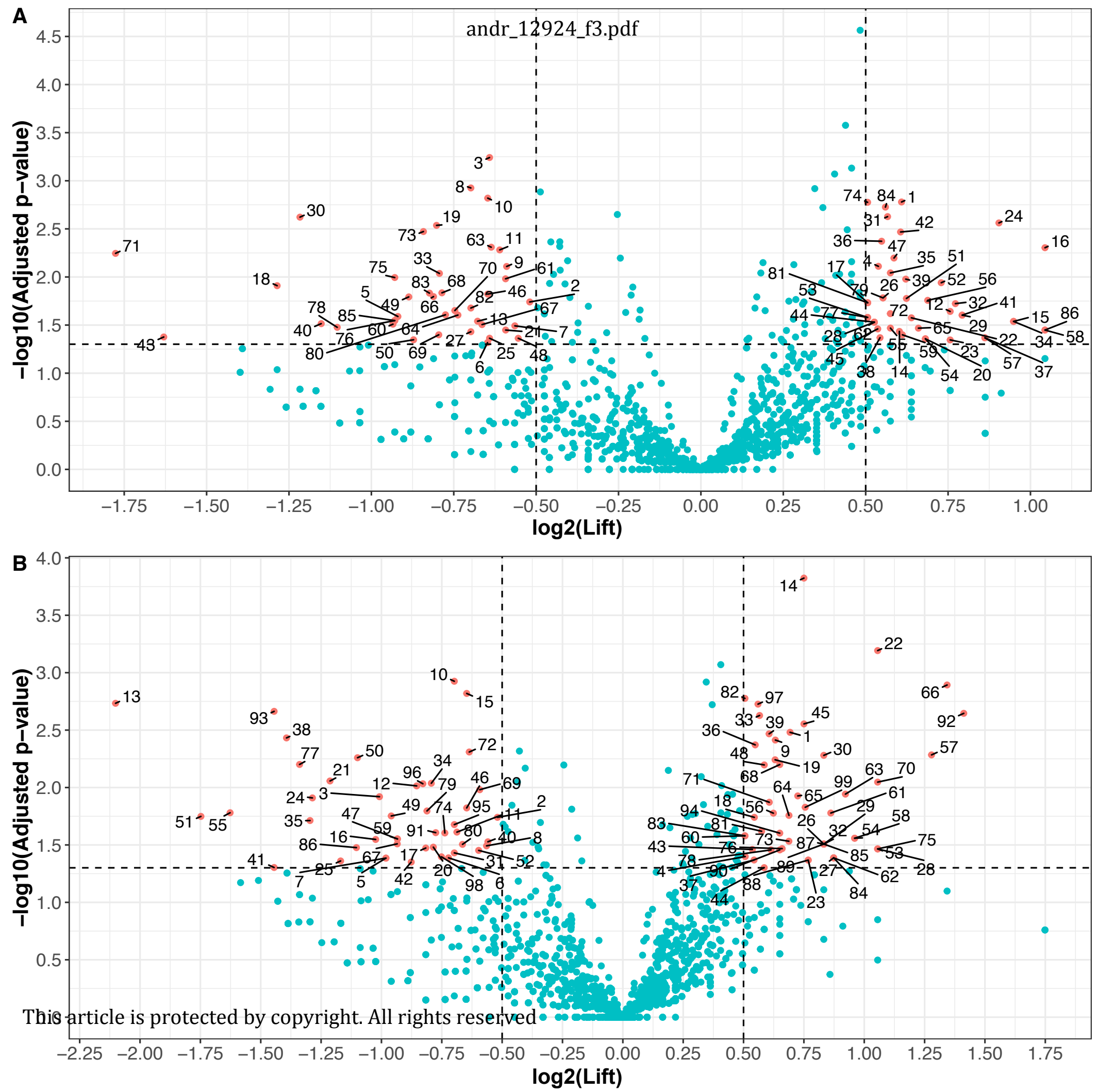